\title{
Down-regulation of NOX4 expression in dorsal root ganglion of spinal cord could alleviate cancer induced bone pain in rats by reducing oxidative stress response
}

Hao Long

The Sixth Affiliated Hospital of Xinjiang Medical University

Hui Zheng

The Sixth Affiliated Hospital of Xinjiang Medical University

Long Ai

The Sixth Affiliated Hospital of Xinjiang Medical University

Kamil Osman

The Second Hospital of Jilin University

Zhigang Liu ( $\square$ liuzhigang_82@163.com )

The Second Hospital of Jilin University

\section{Research}

Keywords: Cancer induced bone pain, NOX4, reactive oxygen species (ROS), dorsal root ganglion of spinal cord

Posted Date: February 19th, 2020

DOI: https://doi.org/10.21203/rs.2.24002/v1

License: (c) (i) This work is licensed under a Creative Commons Attribution 4.0 International License.

Read Full License 


\section{Abstract}

\section{Background}

Cancer-induced bone pain (CIBP) is a common complication in many patients with malignant tumors, and seriously affects their quality of life. Recent studies have implicated reactive oxygen species (ROS) in the perception and regulation of pain through the spinal cord, indicating their possible involvement in CIBP as well. NADPH oxidases (NOX) generate ROS such as $\mathrm{H} 2 \mathrm{O} 2$, superoxide anion, nitric oxide etc. as byproducts of oxidative phosphorylation. In this study, we will analyze the expression levels of NOX4 and its mechanism of action in CIBP.

Material and Methods

A CIBP model was established in rats and NOX4 was knocked down in the dorsal root ganglia via intrathecal injection of specific lentivirus. The downstream effects of NOX4 knockdown were analyzed by RT-PCR, immunofluorescence and Western blotting.

Results

NOX4 was significantly upregulated in the rats with CIBP, and mainly localized in the microglia of the dorsal root ganglion of spinal cord. However, NOX4 knockdown alleviated CIBP by reducing oxidative stress and weakening spinal cord sensitization to pain.

Conclusion

Down-regulation of NOX4 in the dorsal root ganglion of spinal cord can alleviate CIBP by reducing oxidative stress.

\section{Background}

Cancer is one of the major causes of death worldwide. Although novel therapeutic strategies have significantly improved the survival of most patients, the pain caused by cancer seriously affects their life quality $[1,2]$. CIBP refers to the complex and intractable pain caused by bone metastasis of highly invasive malignancies such as breast cancer, prostate cancer, lung cancer etc. The pathogenesis of CIBP is complex and has not yet been fully elucidated. Studies show that sustained stimulation of peripheral nociceptors by secreted tumorigenic, osteolytic and inflammatory factors, as well as periosteal traction or compression of peripheral nerves, blood vessels and other tissues due to tumor invasion sensitizes the peripheral neurons to pain. In addition, central sensitization due to aberrant release of excitatory and inhibitory neurotransmitters and overactivation of corresponding receptors is also closely related to CIBP, although the specific mechanism is still unclear[3-5].

Nicotinamide adenine dinucleotide phosphatase (NADPH) oxidase (NOX) is a group of protease complexes that produce reactive oxygen species (ROS) such as $\mathrm{H}_{2} \mathrm{O}_{2}$, superoxide anion, nitric oxide, etc. 
during oxidative phosphorylation[6, 7]. NOX4 was the first kidney-specific NOX to be discovered, and has since been detected in nerve cells and vascular smooth muscle cells, and in organelles including mitochondria, nuclei and endoplasmic reticulum. Under normal circumstances, the baseline amounts of ROS are neutralized by superoxide dismutase (SOD), catalase (CAT), glutathione peroxidase $\left(\mathrm{GP}_{\mathrm{X}}\right)$ and other antioxidants, which maintain a dynamic balance between ROS generation and elimination[6, 7]. However, chronic and stressful stimuli can trigger a surge in ROS levels that overwhelm the antioxidant system, leading to oxidative stress and a pathological state. Recent studies show that ROS is involved in regulating sensory transmission and its high levels are responsible for nerve injury-related chronic pain. For instance, ligation of the L5 spinal nerves in a rat model of neuropathic pain significantly increased ROS production in the microglia of dorsal root ganglion (DRG)[8]. In addition, Zhou et al.[9] recently showed that the ROS scavenger N-tert-Butyl-a-phenylnitrone (PBN) and tetramethylpiperidine (4-hydroxy2, 2,6,6-tetramethylpiperidine-1-oxyl, Tempol) reduced the mechanical pain in a rodent CIBP model by inhibiting DRG microglial activation. These findings indicate that ROS is likely involved in the perception and regulation of pain through the spinal cord, and therefore a causative factor of CIBP. Accordingly, we hypothesized that down-regulating NOX4 in the DRG tissues would alleviate pain by inhibiting ROS production. To this end, we established a rat model of CIBP and specifically knocked down NOX4 in the DRG neurons through intrathecal lentiviral injection. The changes in pain threshold, ROS production and other related indicators were measured in order to determine the role of NOX4 in CIBP and the underlying mechanisms.

\section{Materials And Methods}

\section{Cell lines and main reagents}

Waiker256 rat breast cancer cells were purchased from Shanghai Cell Bank, Chinese Academy of Sciences. RPMI-1640 and fetal bovine serum (FBS) were purchased from Gibico. RIPA lysate and BCA protein quantification kit were purchased from Invitrogen, and the reverse transcription and real-time quantitative PCR kit from TaKaRa. The NOX4-targeting (RNAi-LV) and negative control (NC-LV) lentiviruses were purchased from Shanghai KeyGen Biotech. Rabbit anti-NOX4 and anti-GAPDH monoclonal antibodies were procured from Abcam, mouse anti-SOD and nNOS from Santa Cruz, goat anti-NMGAR2D and GABAA- 2 from CST company, and the rabbit anti-NOX4, goat anti-NeuN, anti-lba1 and anti-GAFP antibodies from Millipore. Alexa Fluor 488 and Alexa Fluor 555 labeled donkey antigoat/rabbit IgG were purchased from Guangzhou Jingcai Biological, and the HRP-labeled goat antirabbit/mouse IgG, immunocytochemical staining kit and HE staining kit from Wuhan Boster Biological Technology Co. Ltd. The Reactive Oxygen (ROS) Detection Kit was purchased from Nanjing Jiancheng Biological Engineering. All PCR primers were synthesized by Shanghai Biotech.

\section{Intrathecal Catheterization And Lentiviral Injection}


SPF-grade healthy male SD rats weighing $180 \sim 220 \mathrm{~g}$ were purchased from Vital River Company and housed at $22-24{ }^{\circ} \mathrm{C}, 55 \%$ humidity and a $12 \mathrm{~h}$ diurnal cycle, with ad libitum access to food and water. The rats were divided randomly into the sham operated (Sham), untreated CIBP (CIBP), empty vector $(\mathrm{CIBP}+\mathrm{NC}-\mathrm{LV})$ and NOX4 knockdown (CIBP + RNAi-LV) groups ( $\mathrm{n}=8$ in each group) and treated accordingly. All animal experiments were approved by the ethics committee of the Second Hospital of Jilin University, and conducted in strict accordance with the guidelines of the International Association for Study of pain on the use of animals for pain experimental research. The rats were anesthetized with isoflurane ( $3 \%$ for induction and $2 \%$ for maintenance). Subarachnoid catheterization was performed in the lumbosacral region as reported previously[10, 11]. Briefly, a PE-10 catheter was inserted into the subarachnoid space through the cerebello-medullary cistern to a depth of 7-7.5 cm along the direction of the spinal cord towards the intumescentia lumbalis of the tail. A $3-0$ silk thread was then passed through the gap of the catheter to suture the muscle and fix the catheter in position. After excluding rats

with nerve dysfunction or catheter detachment $20 \mathrm{~h}$ after catheterization, the guide tube was injected into the remaining animals with $10 \mathrm{~mL} 2 \%$ lidocaine. Paralysis in the hind limbs confirmed correct positioning of the catheter, following which the rats were injected intraperitoneally with $5 \mathrm{~mL}$ normal saline. On the third day after intrathecal catheterization, $10 \mu \mathrm{L}$ RNAi-LV or NC-LV was injected daily through the intrathecal catheter for 3 consecutive days. The sham-operated animals were injected with an equal volume of normal saline. All rats of the same groups were housed in a single cage after catheterization.

\section{Establishment Of CIBP Model}

Walker 256 cells were maintained in RPMI- 1640 medium containing $10 \% \mathrm{FBS}$ at $37{ }^{\circ} \mathrm{C}$ under $5 \% \mathrm{CO}_{2}$. Viable cells were harvested, re-suspended in PBS at the density of $2 \times 10^{7}$ cells $/ \mathrm{mL}$, and inoculated into the abdominal cavity of rats at the volume of $500 \mu \mathrm{L}$. After one week of conventional feeding, $10 \mathrm{~mL}$ tumor ascites was extracted from each animal and centrifuged at $1000 \mathrm{rpm}$ for $8 \mathrm{~min}$. The pelleted cells were resuspended in PBS at the density of $2 \times 10^{7} / \mathrm{mL}$, and a small aliquot was boiled for 20 min for the Sham group rats. The CIBP model was established as previously described ${ }^{[12]}$. Briefly, the rats were anesthetized (see Sect. 1.2.1) on the third day after lentiviral injection and placed in supine position. The skin of the left hind limb was depilated and disinfected with iodine, and cut longitudinally at the joint of the left knee. The surface of tibial plateau was fully exposed by blunt dissection of the muscle, and the bone was drilled perpendicular to the surface with a No. 5 needle till the bone marrow cavity. The needle was then pulled out, and $10 \mu \mathrm{L}\left(\sim 2 \times 10^{5}\right.$ cells $)$ of the live or dead Walker256 suspension was injected slowly into the cavity using a $25 \mu \mathrm{L}$ microsyringe. The latter was drawn after $30 \mathrm{~s}$, and the injection hole was closed with sterile bone wax. After sterilizing the wound, the muscle and skin layers were sutured.

\section{Analgesic Behavioral Testing}

The calibrated Von-Frey cilia were used to determine the paw withdraw threshold (PWT) of the hind foot on the affected side. As previously reported by Chaplan et al. ${ }^{[12]}$, the rats were acclimatized for $30 \mathrm{~min}$ 
prior to the experiment and the bottom of the third and fourth feet of the right hind foot were stimulated by the tip of Von-Frey cilia. The stimulation intensity was steadily increased from 0 to $40 \mathrm{~g}$ within $20 \mathrm{~s}$. The time taken for the rat to withdraw or move the foot was recorded as the PWT. Paw withdrawal latency (PWL) of the middle and posterior 1/3rd of the right plantar was evaluated in response to thermal pain. The intensity of thermal irradiation was adjusted to $10 \mathrm{~V}$ and $50 \mathrm{~W}$, and the diameter of the stimulated spot was $0.8 \mathrm{~cm}[13,14]$. The reaction time of retracting, adding or lifting the foot, or biting was recorded as PWL. All measurements were taken between 8:00 a.m. to 12:00 noon by the same technicians, and each animal was stimulated thrice at 3 min intervals. The PWT and PWL scores were evaluated before and on days $0,3,7,14$ and 21 post-intrathecal catheterization.

\section{Histopathology}

The rats were deeply anesthetized by intraperitoneal injection of $10 \%$ chloral hydrate on the 21 st day after CIBP induction and dissected. The affected tibia was removed and decalcified in 15\% EDTA-2Na for 3 weeks, with the solution replaced weekly. After washing with PBS, the decalcified tibia was embedded in paraffin and sectioned. The tissue sections were dewaxed, dehydrated through an alcohol gradient, and stained by HE before microscopic examination.

\section{DRG Harvest And Immunohistochemistry (IHC)}

The rats were anesthetized as above, and the dorsal region $2 \mathrm{~cm}$ on either side of the median line as well as both hind limbs was depilated. The animals were dissected along the median dorsal line from the tail to the upper part of the hind limbs, and the subcutaneous tissues and muscles were separated to expose the spine. The L4-6 section on the left side of the spinal cord harboring the DRG tissue was extracted, washed with chilled PBS and frozen in liquid nitrogen for molecular assays. For IHC, the chest wall of the anesthetized rats was opened to expose the heart, and $10 \mathrm{~mL}$ normal saline was injected into the aorta through the left ventricle following exsanguination. After the saline wash, the animals were perfused with $4 \%$ polyformaldehyde, and the DRG tissues were removed and fixed in $4 \%$ paraformaldehyde for $2 \mathrm{~h}$. The fixed tissues were rinsed with PBS, dehydrated in $30 \%$ sucrose solution, embedded in OTC, and frozen at $-20{ }^{\circ} \mathrm{C}$ for cryo-sectioning. The sections were rinsed thrice with PBS, immersed in blocking solution for $1 \mathrm{~h}$ at room temperature, and incubated overnight with anti-NOX4 (1:500) and anti-NeuN (1:200), anti-GFAP (1:300) or anti-lba $1(1: 300)$ antibodies at $4{ }^{\circ} \mathrm{C}$. After washing thrice with PBS, the sections were incubated with Alexa Fluor 488 and Alexa Fluor 555 labeled secondary antibodies for $2 \mathrm{~h}$ at room temperature. Following a final wash cycle, the stained sections were observed and imaged under a confocal microscope.

\section{Detection Of In Situ $\mathrm{H}_{2} \mathrm{O}_{2}$}


The DRG tissues were washed thrice with chilled PBS and homogenized in RIPA lysis buffer. The lysates were centrifuged at $12,000 \mathrm{rpm}$ at $4{ }^{\circ} \mathrm{C}$ for $5 \mathrm{~min}$ and the supernatants were aspirated. The concentration of $\mathrm{H}_{2} \mathrm{O}_{2}$ was measured using the specific kit as per the manufacturer's instructions.

\section{Real-time Fluorescence Quantitative Pcr (RT-PCR)}

Total RNA was extracted from the DRG tissues using Trizol, and the purity and concentration were detected using a spectrophotometer. RNA was reverse transcribed into cDNA using a reverse transcription kit, and RT-PCR was performed according to the kit instructions and reaction conditions standardized by preliminary experiments. The RT-PCR primer sequences are as follows:

NOX4-F:5'-TCTGGAAAACCTTCCTGCTG-3', NOX4-R:5'-CCGGCACATAGGTAAAAGGA-3';NMGAR2D-F: 5'GCCCTGGCAGTGTCTTAG-3', NMGAR2D-R:5'-CAGTGCGTGTCGTGGAG-5'; GABAA-ү2F:5'GCAGCAGCGGCTTCACA-3', GABAA-ү2-R:ACATCCAAACAGGAGCGTCAT; GAPDH-F:5' AGAAGGCTGGGGCTCATTTG 3',GAPDH-: 5' AGGGGCCATCCACAGTCTTC 3'. GAPDH was used as the internal reference, and the expression levels of the target RNA was analyzed by the 2- $\Delta \Delta \mathrm{Ct}$ method.

\section{Western Blotting}

The DRG tissues were washed thrice with chilled PBS, and homogenized with RIPA lysis buffer supplemented with protease inhibitor. Following quantification with the BCA kit, $35 \mu \mathrm{g}$ of each protein sample was mixed with $5 \times$ loading buffer at the ratio of 1:4, denatured by boiling for $10 \mathrm{~min}$, and separated by polyacrylamide gel electrophoresis (SDS-PAGE). The protein bands were transferred to PVDF membrane by wet rotation method and blocked with $5 \%$ skim milk at room temperature for $2 \mathrm{~h}$. The blots were then incubated overnight with primary antibodies against SOD (1: 500), nNOS (1: 500), GABAAY2 (1: 300), NMDAR2D (1: 500) and GAPDH (1: 1500) at $4{ }^{\circ} \mathrm{C}$ with constant shaking. After washing thrice with TBST solution for 5 min each time, the membranes were incubated with HRP-labeled secondary antibody (1: 5000) for $1 \mathrm{~h}$ at room temperature, and washed again as described. The positive bands were developed using an ECL reagent and exposed in a gel imager. The gray values of the bands were measured by Image $\mathrm{J}$ software, and the ratio of the target protein to the internal reference GAPDH was calculated. Three independent experiments were performed.

\section{Statistical analysis}

SPSS 19.0 and GraphPad Prism 5.0 were used for statistical analysis and all data were expressed as 'X \pm S. Two groups were compared using the independent sample t-test, and multiple groups by one-way ANOVA and Dunnett's or Bonferroni's ad-hoc test. The significance test level was $\alpha=0.05$, and $P<0.05$ was considered statistically significant.

\section{Results}




\section{Successful construction of ClBP model}

The induction of CIBP was verified in terms of pathological and behavioral parameters. As shown in Fig. 1a, the tibias of the sham-operated rats were intact with a clear boundary between the bone tissue and cavity, and abundant bone marrow. In contrast, inoculation of tumor cells resulted in visible deterioration of the tissue microstructure and replacement of the bone marrow cells with tumor cells within 7 days, which progressed to severe destruction of the bone matrix and trabeculae, obliteration of the boundary between tissue and marrow cavity, and excessive tumor cell proliferation in the bone marrow by the 14th and 21 st day (Fig. 1a-d). The basal mechanical and thermal pain thresholds were similar across all groups. However, the pain threshold of the CIBP rats decreased significantly from day 7 post-inoculation, and sensitivity to pain gradually increased over the progression of disease. In contrast, the pain threshold of the sham-operated rats was relatively stable (Fig. 1e, f). Taken together, the CIBP model was successfully established.

\section{NOX4 is upregulated in the DRG microglia upon CIBP induction}

NOX4 mRNA and protein expression increased significantly in the DRG tissues of the CIBP modeled rats compared to the sham-operated animals, and showed a time-dependent upregulation with the progression of the disease (Fig. 2a, b). Furthermore, co-staining of the DRG tissues with NeuN, GFAP or Iba 1 and NOX4 indicated that the latter was primarily localized to the microglia as opposed to the neurons and astrocytes (Fig. 2c-k).

\section{NOX4 Knockdown Significantly Alleviated CIBP}

To further elucidate the biological relevance of NOX4 in CIBP, we knocked down the gene in the DRG tissues via intrathecal lentiviral injection. As shown in Fig. 3a-b, NOX4 mRNA and protein levels were significantly downregulated in the rats infected with RNAi-LV compared to the Sham and NC-LV groups. DRG-specific knockdown of NOX4 significantly alleviated the mechanical and thermal pain sensitivity in the rats, thereby underscoring the causative role of NOX4 in CIBP (Fig. 3c, d).

\section{NOX4 Knockdown Alleviated Oxidative Stress In DRG Tissue}

NOX4 significantly decreased ROS levels in DRG tissue (Fig. 4a), and concomitantly increased that of the antioxidant enzyme SOD (Fig. 4b). ROS like the superoxide anions combine with NO to form nitrate, which inactivates SOD. In nerve tissues, NO is mainly produced by neuronal nitric oxide synthase (nNOS), which also plays an important role in neuropathic pain ${ }^{[15]}$. However, NO is difficult to detect in tissues and 
cells. Nevertheless, we found that down-regulation of NOX4 expression significantly inhibited nNOS in DRG tissues (Fig. 4b). Taken together, NOX4 promotes oxidative stress in the DRG microglia by enhancing ROS production and inhibiting SOD, which can be reversed by NOX4 knockdown.

\section{NOX4 Knockdown Alters Neurotransmitter Receptor Activation}

Since ROS accumulation can alter the activation status of the glutamate and gamma-aminobutyric acid (GABA) neurotransmitter receptors, we next analyzed the effect of NOX4 knockdown on these receptors. NR2D, the major subtype of the excitatory receptor NMDAR, was significantly elevated in the rats with $\mathrm{CIBP}$, whereas the inhibitory receptor GABAA-y2 was significantly decreased. Down-regulation of NOX4 reduced the activation levels of NR2D and significantly increased that of GABAA- $\gamma 2$ (Fig. 5a). Similar results were seen with the mRNA and total protein expression levels of both receptors in the control and NOX4-knockdown DRG tissues (Fig. 5b-c).

\section{Discussion}

CIBP is generally the earliest palpable symptom in various malignancies, and most patients seeking treatment for the pain are already in the advanced stages of cancer. However, the pathogenesis of CIBP is complex and ambiguous, leading to unsatisfactory clinical outcomes. Therefore, it is essential to identify the molecular mechanisms underlying CIBP in order to improve treatment strategies[16].

Peripheral sensitization due to continuous stimulation by various inflammatory cytokines, the acidic tumor-bone microenvironment and altered ROS levels, and central sensitization caused by synaptic plasticity are closely related to the occurrence of $\mathrm{CIBP}^{[17-19]}$. Direct injection of cancer cells into the bone marrow cavity can simulate the clinical characteristics of CIBP and therefore help explore its molecular mechanisms. Intrathecal injection of rat breast cancer cells into the tibia marrow cavity of adult female rats resulted in mechanical and thermal hyperalgesia within a week, and increased pain sensitivity over a period of 3 weeks. This corresponded to progressive deterioration of the bone trabeculae and the gradual colonization of the marrow cavity with highly differentiated tumor cells. These results confirmed that CIBP was successfully established in the animal model.

The NOX family of proteases are the major catalysts of ROS generation and oxidative stress. NOX4 is distinct from other NOX isomers on account of its tissue-specific expression and unique molecular structure. In addition, while activation of NOX1-3 depends on the phosphorylation of cellular subunits and that of NOX5 on $\mathrm{Ca}^{2+}$ signaling, NOX4 harbors an intrinsic activated dehydrogenase domain that can generate ROS through the electron transfer from NADPH to FDA. However, due to the highly conserved histidine residue in its third extracellular ring, NOX4 could induce superoxide to spontaneously disproportionate into $\mathrm{H}_{2} \mathrm{O}_{2}$ in the enzyme, and therefore the ROS produced by NOX4 is mainly $\mathrm{H} 2 \mathrm{O} 2[20$, 21]. In this study, we detected a significant upregulation of NOX4 mRNA and protein expression during 
disease progression. In addition, NOX4 was mainly localized in the microglia of DRG tissues. Microglia are the resident macrophages of the central nervous system (CNS), and account for more than $5 \%$ of the nerve cells in the brain. When the CNS is subjected to pessimal stimulation, the microglia are rapidly activated and release effectors like ROS, NO and inflammatory factors (e.g. TNF - $a$, IL-1 $\beta$ and COX-2), which can significantly damage peripheral nerve cells, especially the neurons. Recent studies show that the interaction between neurons and microglia is crucial to central sensitization and the feeling of pain $[20,21]$. To elucidate the mechanistic role of microglial NOX4 in CIBP, we specifically knocked it down in the DRG tissues, and observed that the animals were significantly sensitized to both mechanical and thermal pain. These preliminary results indicate that NOX4 is a causative factor in CIBP and therefore can be targeted to alleviate bone pain in cancer patients.

The CNS is metabolically demanding due to the high proportion of unsaturated fatty acids, which increases its susceptibility to oxidative stress. The neurons in particular are highly sensitive to ROSinduced injury, which is the mechanistic basis of neurodegenerative disorders like Parkinson's disease, Alzheimer's disease, stroke and other neurological lesions ${ }^{[22]}$. Hassler et al.[8] found that Ros promoted chronic neuralgia after spinal cord injury, and its neutralization significantly relieved the intensity of pain. Little et al. ${ }^{[23]}$ have postulated that ROS and NO are key players in the development of pain via central sensitization. $\mathrm{H}_{2} \mathrm{O}_{2}$ plays an important role in pain perception and regulation as a diffusive signaling molecule. Indeed, $\mathrm{H}_{2} \mathrm{O}_{2}$ levels increased significantly in the DRG of a rat neuropathic pain model established via spinal cord transection, and induced neuronal apoptosis through the PI3K/AKt signaling pathway. SOD is closely related to the perception and regulation of pain. Clinical studies show that SOD levels are significantly lower in patients with chronic migraine compared to healthy controls. Consistent with this, the CIBP rats also expressed lower levels of SOD compared to the sham-operated animals. This could be due to the accumulation of large amounts of ROS that combines with nitric oxide to form peroxynitrite, which in turn reduces the content of SOD through nitrification. NOX4 knockdown in the DRG significantly upregulated SOD, likely by inhibiting the production of $\mathrm{H}_{2} \mathrm{O}_{2}$ and nNOS.

Glutamate and $y$-aminobutyric acid (GABA) are respectively the major excitatory and inhibitory neurotransmitters in the CNS, and play a key role in inflammation-related pain as well as CIBP[24]. The considerable increase in ROS levels in the bone tumor microenvironment leads to the production and secretion of excessive amounts of glutamic acid by the tumor cells. The accumulation of glutamate increases the excitability of afferent nerves by activating N-methyl-D-aspartic acid (NMDA) receptors on peripheral nerve endings, which in turn alters synaptic plasticity in the DRG and enhances pain responsiveness and central sensitization ${ }^{[24]}$. In fact, direct injection of ROS into the spinal cord of rats with neuropathic pain not only aggravated the pain response, but also significantly reduced the expression of GABA. Introducing the ROS scavenger PBN significantly alleviate the above ${ }^{[25]}$. Consistent with this, knocking down NOX4 in the CIBP model significantly downregulated the excitatory receptor NMDAR in the DRG tissue and upregulated the inhibitory receptor GABAA- $\gamma 2$.

\section{Conclusion}


NOX4 is significantly upregulated in the DRG microglia of rats with CIBP, and its inactivation alleviated pain by reducing oxidative stress and weakening spinal cord sensitization. Thus, NOX4 is a potential therapeutic target in CIBP and its role needs to be investigated further, along with the additional mechanisms of CIBP.

\section{Abbreviations}

CIBP, Cancer-induced bone pain; ROS: reactive oxygen species; NOX: NADPH oxidases; CNS: central nervous system; GABA: Glutamate and $y$-aminobutyric acid; NMDA: N-methyl-D-aspartic acid

\section{Declarations}

Ethics approval and consent to participate

All animal experiments were approved by the ethics committee of the Second Hospital of Jilin University, and conducted in strict accordance with the guidelines of the International Association for Study of pain on the use of animals for pain experimental research.

Availability of data and materials

All data are available in the included figures.

Competing interests

The authors declare that they have no conflict of interests.

Acknowledgements

None.

Consent for publication

None.

Funding

This work is supported by Xinjiang Uygur Autonomous Region Natural Science Foundation Project (2017D01C257)

Authors' contributions

Conceptualization: HL, ZL; Methodology: HL, HZ, ZL; Software: HZ, LA; Supervision: ZL; Writing original draft: HL, KO, ZL; All authors read and approved the final manuscript.

\section{References}


1. Mantyh PW. Cancer pain and its impact on diagnosis, survival and quality of life. Nature Reviews Neuroscience 2006; 7(10): 797-809.https://doi.org/10.1038/nrn1914

2. Mantyh PW. Mechanisms that drive bone pain across the lifespan. British journal of clinical pharmacology 2019; 85(6): 1103-1113.https://doi.org/10.1111/bcp.13801

3. Ryu HK, Baek Y-H, Park Y-C, Seo B-K. Current studies of acupuncture in cancer-induced bone pain animal models. Evidence-Based Complementary and Alternative Medicine 2014; 2014.https://doi.org/10.1155/2014/191347

4. Fu Q, Shi D, Zhou Y, Zheng H, Xiang H, Tian X, Gao F, Manyande A, Cao F, Tian Y. MHC-I promotes apoptosis of GABAergic interneurons in the spinal dorsal horn and contributes to cancer induced bone pain. Experimental neurology 2016; 286: 1220.https://doi.org/10.1016/j.expneurol.2016.09.002

5. Yao P, Ding Y, Han Z, Mu Y, Hong T, Zhu Y, Li H. Suppression of asparaginyl endopeptidase attenuates breast cancer-induced bone pain through inhibition of neurotrophin receptors. Molecular pain 2017; 13: 1744806917708127. https://doi.org/10.1177/1744806917708127

6. Hollins F, Sutcliffe A, Gomez E, Berair R, Russell R, Szyndralewiez C, Saunders R, Brightling C. Airway smooth muscle NOX4 is upregulated and modulates ROS generation in COPD. Respiratory research 2016; 17(1): 84.https://doi.org/10.1186/s12931-016-0403-y

7. Im Y, Jee M, Choi J, Cho H, Kwon O, Kang S. Molecular targeting of NOX4 for neuropathic pain after traumatic injury of the spinal cord. Cell death \& disease 2012; 3(11): e426e426.https://doi.org/10.1038/cddis.2012.168

8. Hassler SN, Johnson KM, Hulsebosch CE. Reactive oxygen species and lipid peroxidation inhibitors reduce mechanical sensitivity in a chronic neuropathic pain model of spinal cord injury in rats. Journal of neurochemistry 2014; 131(4): 413-417.https://doi.org/10.1111/jnc.12830

9. Zhou Y-Q, Liu D-Q, Chen S-P, Sun J, Zhou X-R, Rittner H, Mei W, Tian Y-K, Zhang H-X, Chen F. Reactive oxygen species scavengers ameliorate mechanical allodynia in a rat model of cancer-induced bone pain. Redox biology 2018; 14: 391-397.https://doi.org/10.1016/j.redox.2017.10.011

10. Liu S, Yang J, Wang L, Jiang M, Qiu Q, Ma Z, Liu L, Li C, Ren C, Zhou J. Tibia tumor-induced cancer pain involves spinal p38 mitogen-activated protein kinase activation via TLR4-dependent mechanisms. Brain research 2010; 1346: 213-223.https://doi.org/10.1016/j.brainres.2010.05.014

11. Yaksh TL, Rudy TA. Chronic catheterization of the spinal subarachnoid space. Physiology \& behavior 1976.https://doi.org/10.1016/0031-9384(76)90029-9

12. Tong Z, Luo W, Wang Y, Yang F, Han Y, Li H, Luo H, Duan B, Xu T, Maoying Q. Tumor tissue-derived formaldehyde and acidic microenvironment synergistically induce bone cancer pain. PloS one 2010; 5(4).https://doi.org/10.1371/journal.pone.0010234

13. Hargreaves K, Dubner R, Brown F, Flores C, Joris J. A new and sensitive method for measuring thermal nociception in cutaneous hyperalgesia. Pain 1988; 32(1): 77-

88.https://doi.org/10.1016/0304-3959(88)90026-7

Page 11/16 
14. Malmquist NA, Moss TA, Mecheri S, Scherf A, Fuchter MJ. Small-molecule histone methyltransferase inhibitors display rapid antimalarial activity against all blood stage forms in Plasmodium falciparum. Proceedings of the National Academy of Sciences 2012; 109(41): 1670816713.https://doi.org/10.1073/pnas.1205414109

15. Demir IE, Heinrich T, Carty DG, Saricaoglu ÖC, Klauss S, Teller S, Kehl T, Reyes CM, Tieftrunk E, Lazarou M. Targeting nNOS ameliorates the severe neuropathic pain due to chronic pancreatitis. EBioMedicine 2019; 46: 431-443.https://doi.org/10.1016/j.ebiom.2019.07.055

16. Sindhi V, Erdek M. Interventional treatments for metastatic bone cancer pain. Pain management 2019; 9(3): 307-315.https://doi.org/10.2217/pmt-2018-0073

17. Yoneda T, Hiasa M, Nagata $Y$, Okui T, White FA. Acidic microenvironment and bone pain in cancercolonized bone. BoneKEy reports 2015; 4.https://doi.org/ 10.1038/bonekey.2015.58

18. Liang Y, Du J-Y, Fang J-F, Fang R-Y, Zhou J, Shao X-M, Jiang Y-L, Chen Y-T, Fang J-Q. Alleviating mechanical allodynia and modulating cellular immunity contribute to electroacupuncture's dual effect on bone cancer pain. Integrative cancer therapies 2018; 17(2): 401410.https://doi.org/10.1177/1534735417728335

19. Dai WL, Yan B, Jiang N, Wu JJ, Liu XF, Liu JH, Yu BY. Simultaneous inhibition of NMDA and mGlu1/5 receptors by levo-corydalmine in rat spinal cord attenuates bone cancer pain. International journal of cancer 2017; 141(4): 805-815.https://doi.org/10.1002/ijc.30780

20. Wang J, Liu Y, Shen H, Li H, Wang Z, Chen G, Nox2 and Nox4 participate in ROS-induced neuronal apoptosis and brain injury during ischemia-reperfusion in rats, in Subarachnoid Hemorrhage. 2020, Springer, 47-54.

21. Wiegman CH, Michaeloudes C, Haji G, Narang P, Clarke CJ, Russell KE, Bao W, Pavlidis S, Barnes PJ, Kanerva J. Oxidative stress-induced mitochondrial dysfunction drives inflammation and airway smooth muscle remodeling in patients with chronic obstructive pulmonary disease. Journal of Allergy and Clinical Immunology 2015; 136(3): 769-780.https://doi.org/10.1016/j.jaci.2015.01.046

22. Maes M, Galecki P, Chang YS, Berk M. A review on the oxidative and nitrosative stress (O\&NS) pathways in major depression and their possible contribution to the (neuro) degenerative processes in that illness. Progress in Neuro-Psychopharmacology and Biological Psychiatry 2011; 35(3): 676692.https://doi.org/10.1016/j.pnpbp.2010.05.004

23. Little JW, Doyle T, Salvemini D. Reactive nitroxidative species and nociceptive processing: determining the roles for nitric oxide, superoxide, and peroxynitrite in pain. Amino acids 2012; 42(1): 75-94.https://doi.org/10.1007/s00726-010-0633-0

24. Ungard RG, Seidlitz EP, Singh G. Inhibition of breast cancer-cell glutamate release with sulfasalazine limits cancer-induced bone pain. PAIN® 2014; 155(1): 28-

36.https://doi.org/10.1016/j.pain.2013.08.030

25. Yowtak J, Lee KY, Kim HY, Wang J, Kim HK, Chung K, Chung JM. Reactive oxygen species contribute to neuropathic pain by reducing spinal GABA release. Pain ${ }^{8} 2011 ; 152(4)$ : 844852.https://doi.org/10.1016/j.pain.2010.12.034 
Figures

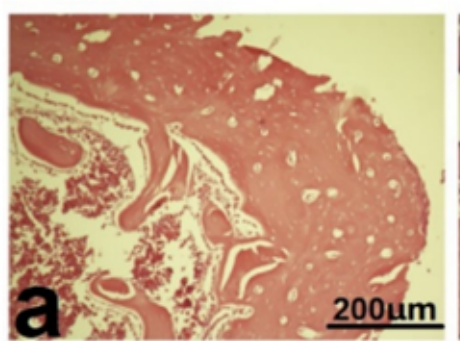

e

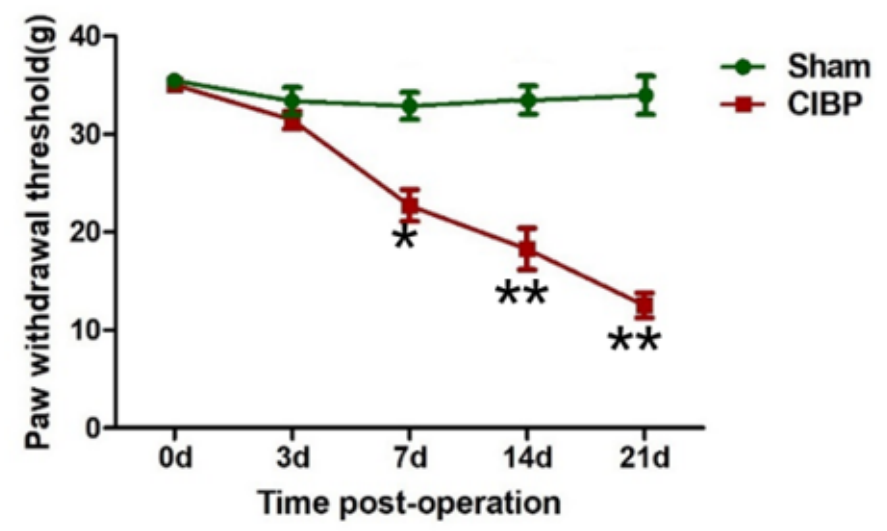

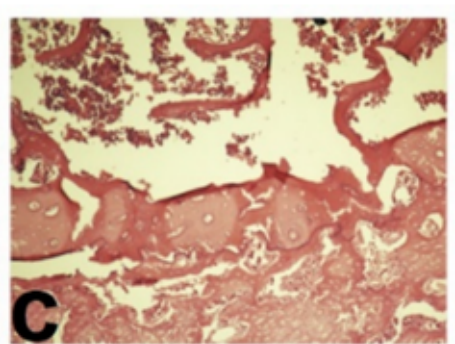

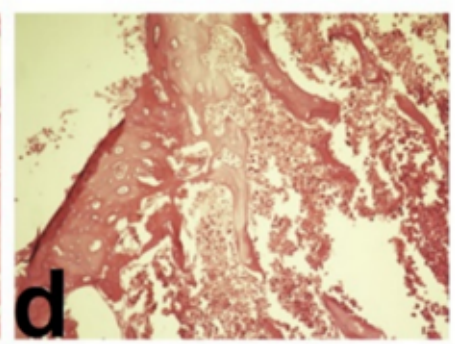

f

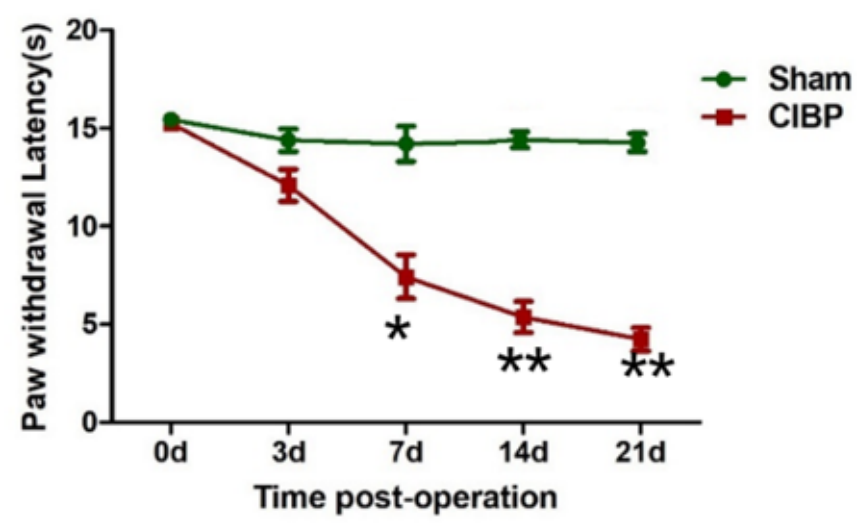

Figure 1

Establishment of CIBP model. a-d: Bone structure of the sham-operated and tumor-bearing rats on days 7 , 14 and 21 after tumor inoculation. e-f: The mechanical (e) and thermal (f) withdrawal thresholds of the sham-operated and tumor-bearing rats at the indicated time points. ${ }^{*} \otimes \square 0.05,{ }^{*} \mathrm{P} \otimes 0.01$ compared to the Sham group. 
a

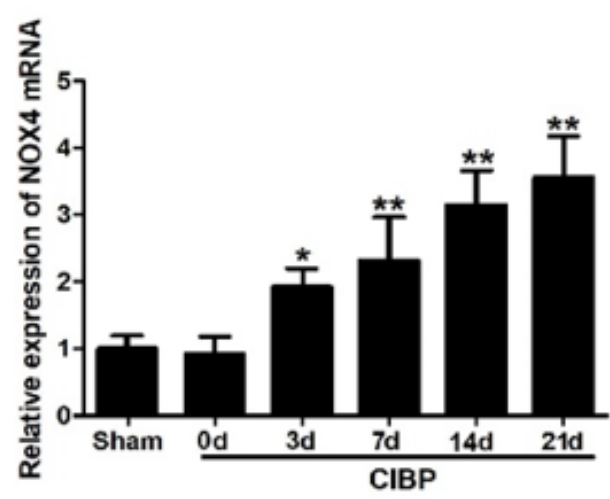

b

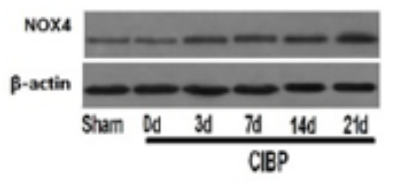

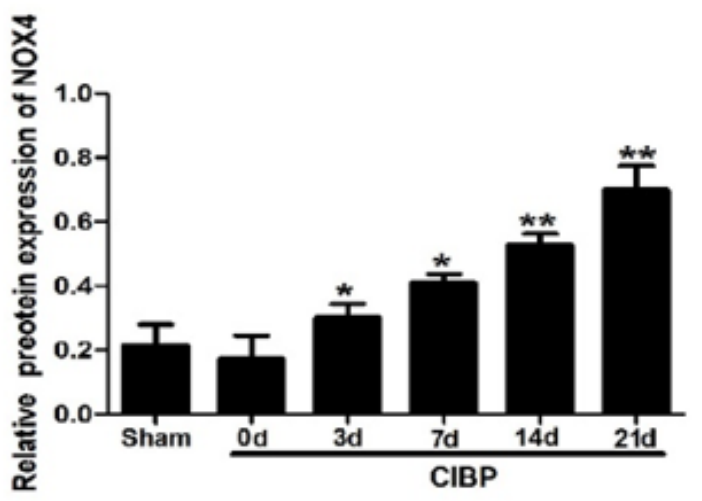

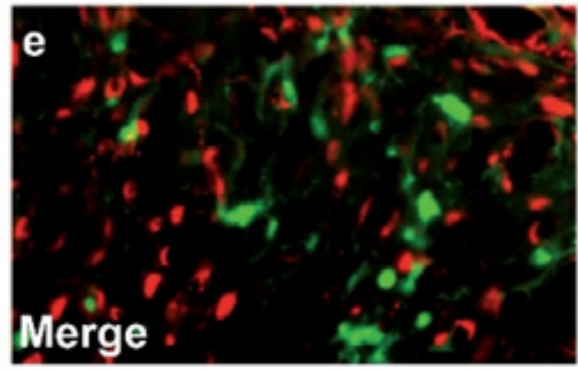

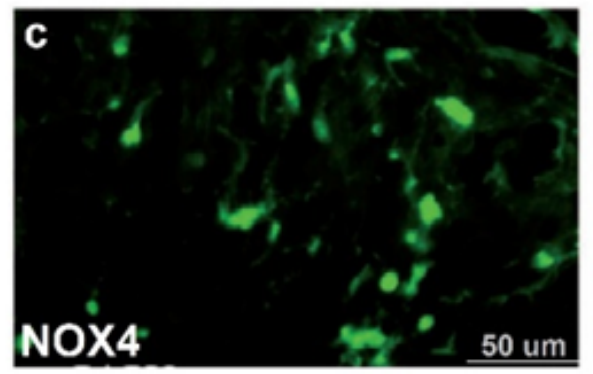
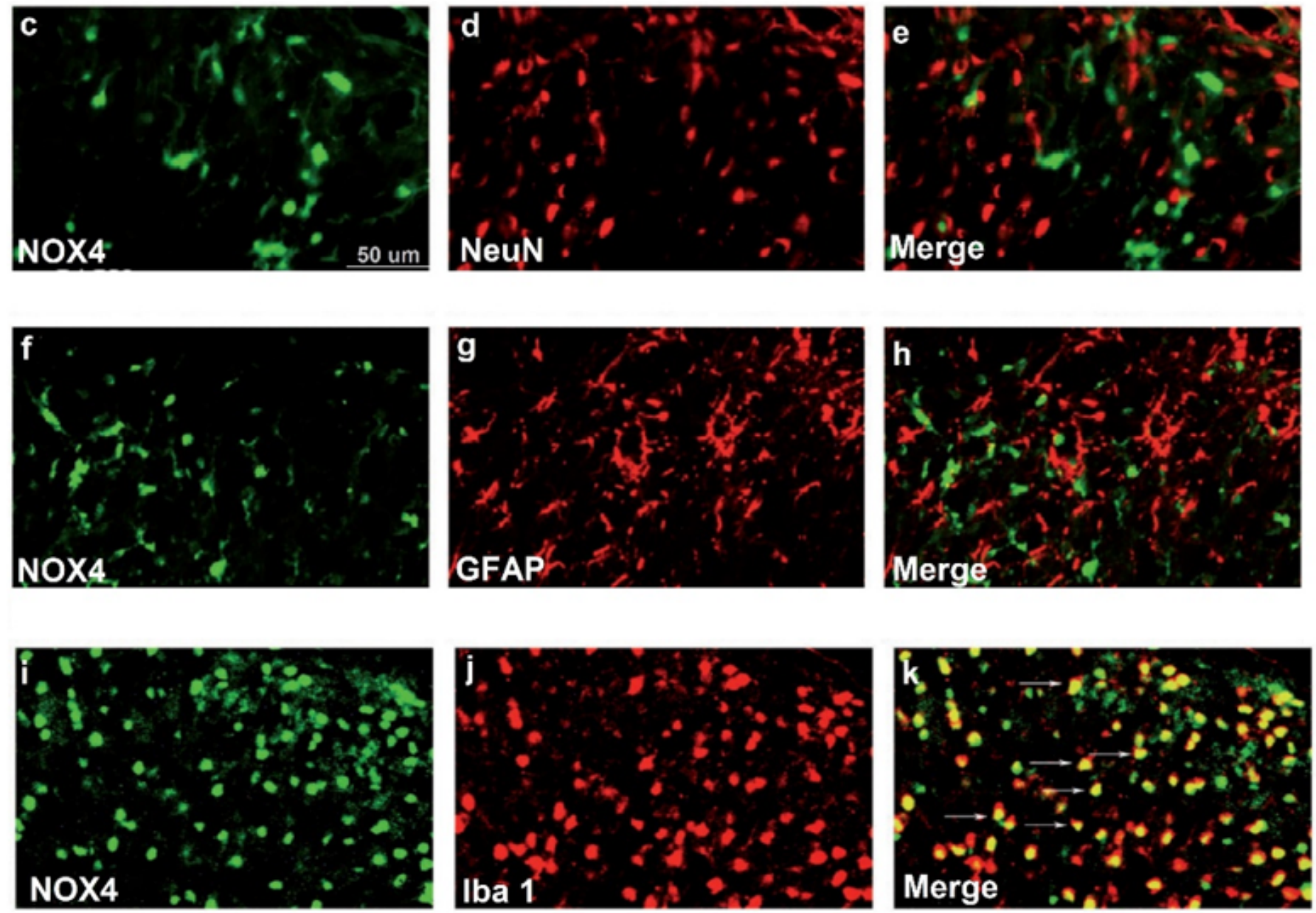

Figure 2

The expression and cellular localization of NOX4 in DRG tissues. a-b: NOX4 mRNA (a) and protein (b) levels in the DRG of Sham and CIBP group rats; c-k: Representative immunofluorescence images showing co-staining of NOX4 (green) with NeuN+ (red) neurons, GFAP+ (red) astrocytes or lba1+ (red) microglia in the DRG tissue of the affected side on the 21 st day after tumor inoculation. Compared with Sham group, ${ }^{*} \mathrm{P}<0.05, * * \mathrm{P}<0.01$. 
a

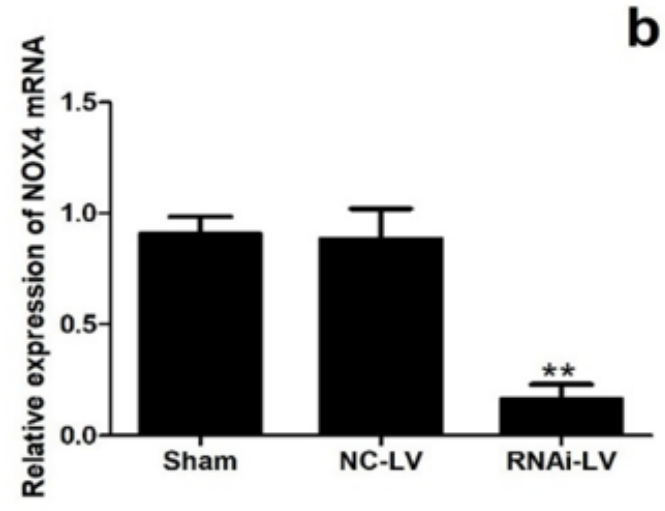

b
C

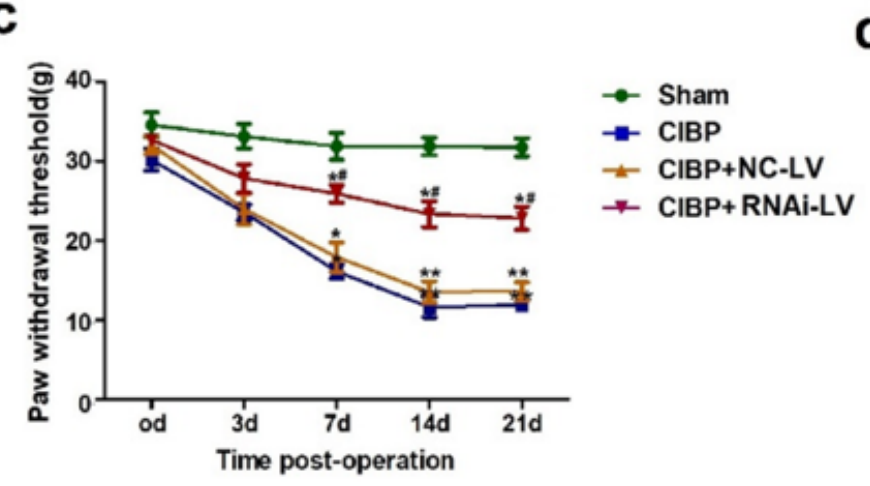

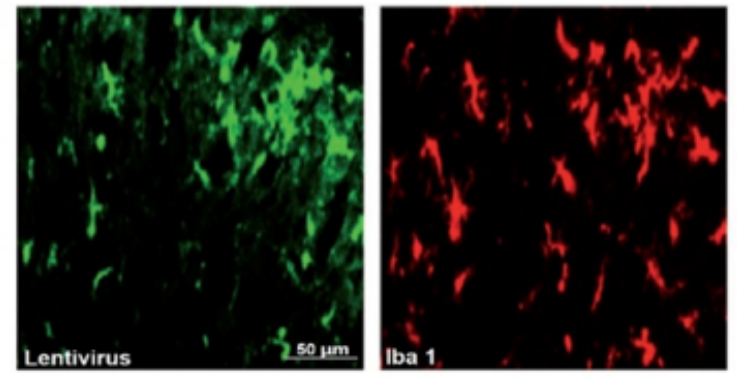

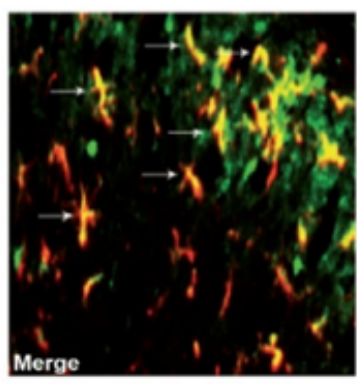

d

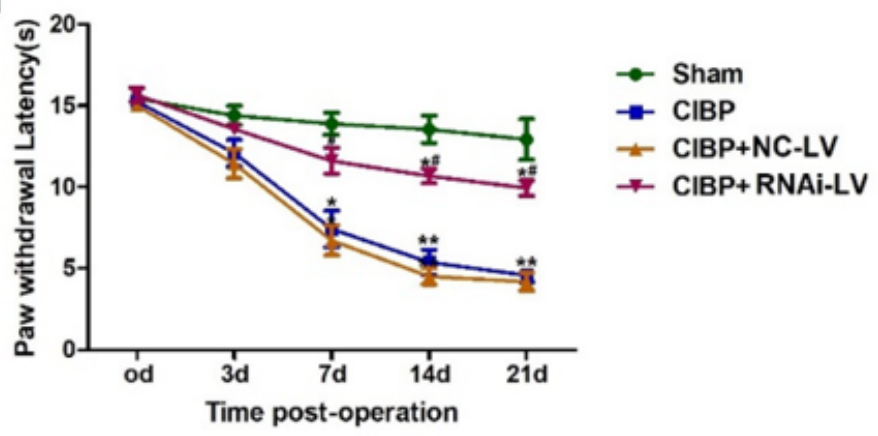

Figure 3

Down-regulation of NOX4 expression significantly alleviated CIBP in rats. a: NOX4 mRNA levels in the DRG tissues; b: Immunofluorescence images showing in situ NOX4 expression in the microglia of Sham, CIBP + RNAi-LV and CIBP + NC-LV groups; (c-d): Mechanical (c) and thermal (d) withdrawal threshold in the Sham and CIBP model rats infected with the indicated lentiviral constructs. Compared with Sham group, * $p<0.05$, ** $p<0.05$; compared with CIBP group, \#p <0.05.

a

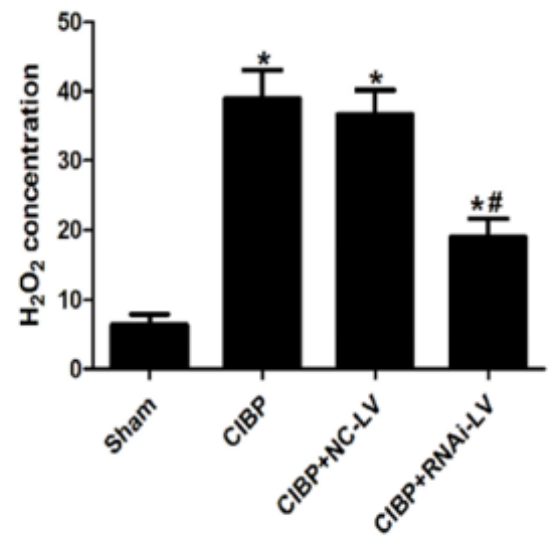

b

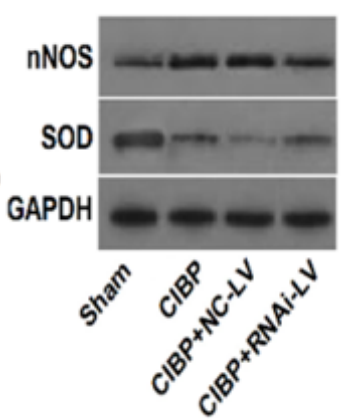

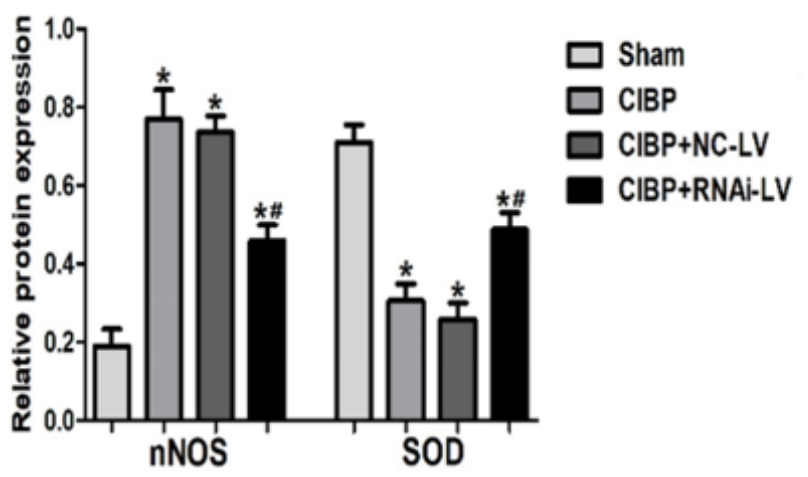

Figure 4 
NOX4 knockdown alleviates oxidative stress in DRG tissue. a: H2O2 levels in the DRG tissues of the different groups; b: Immunoblot showing expression levels of nNOS and SOD in DRG tissue of different groups. Compared with Sham group, ${ }^{*} \mathrm{P}<0.05$, ${ }^{\star \star} \mathrm{P}<0.05$; compared with CIBP group, $\# \mathrm{P}<0.05$.

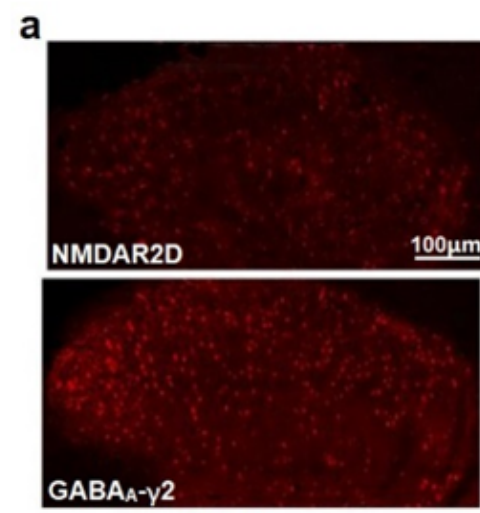

Sham
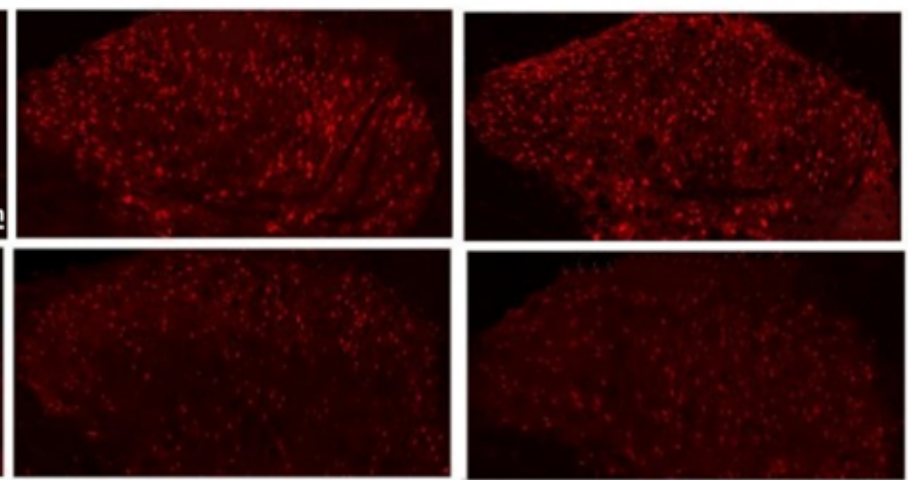

CIBP

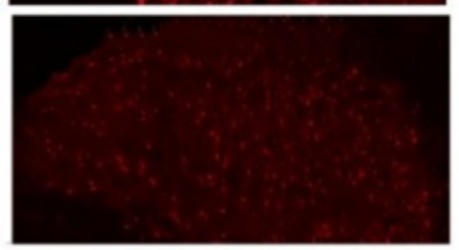

CIBP+NC-LV
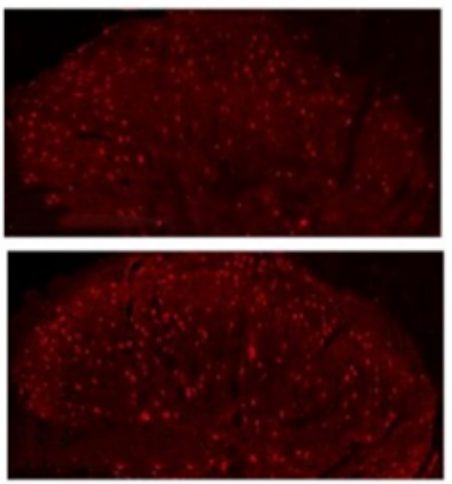

CIBP+RNAi-LV b

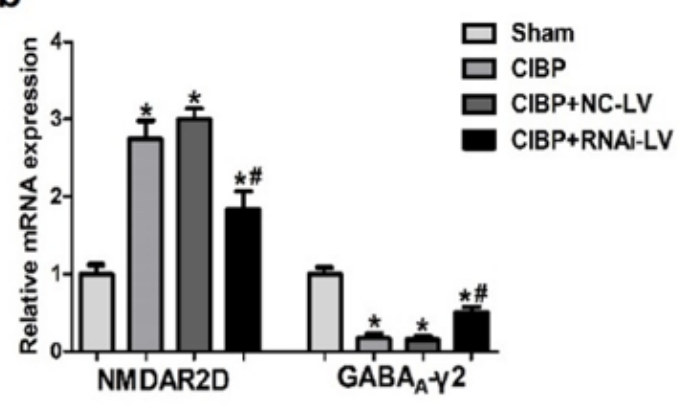

C

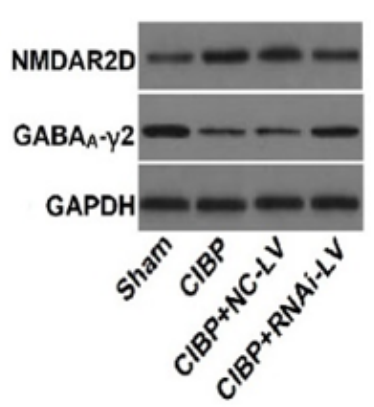

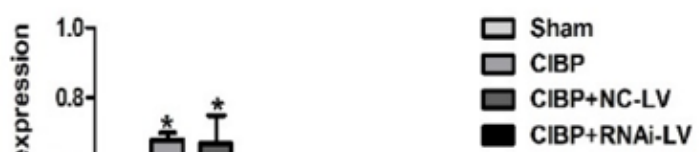

$\square$ Sham
$\square$ CIBP
$\square$ CIBP+NC-LV
CIBP+RNAi-LV

\section{Figure 5}

The effect of NOX4 knockdown on the activation of neurotransmitter receptors in DRG tissue. a:

Representative immunofluorescence images showing in situ expression of NMDAR2D and GABAA- $y 2$ in the DRG tissue of different groups; $b$ : NMDAR2D and GABAA- 2 mRNA levels in the above; $c$ : Immunoblot showing NMDAR2D and GABAA- $\gamma 2$ protein levels in the above. Compared with Sham group, ${ }^{\star} p<0.05,{ }^{\star \star} p$ $<0.05$; compared with CIBP group, \#p <0.05 